\title{
EL NIÑO SIN DERECHOS
}

\section{INGER SOPHIE BENSON}

Hogskolen I Bodo (Noruega).

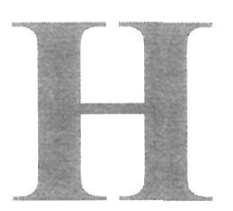

\section{INTRODUCCIÓN}

e escrito un libro sobre un niño que vivió once años y ocho meses en un hospital general. El título es El niño sin derechos. Este niño estuvo ingresado como paciente desde su nacimiento hasta esa edad. Cuando cumplió once años y ocho meses se mudó a una vivienda propia, cerca de la casa de su madre. Este niño nunca ha vivido en una familia.

Este niño no ha estado en el hospital a causa de su enfermedad, sino que se quedó en él porque ni su padre ni su madre estaban en condiciones de cuidarle. El niño nació con los pulmones subdesarrollados, solamente. Mi libro es una descripción sobre la vida del niño durante su estancia en el hospital.

¿Por qué ha pasado esto? Y ¿por qué el niño nunca tuvo la posibilidad de crecer en una familia? Mi libro es también una descripción de mi lucha por conseguir una familia para el niño. Yo luché durante tres años y medio para lograrlo y no lo logré.

"Los niños crecen en familias, no en instituciones". Mi libro es también una descripción sobre los prejuicios frente a las discapacidades. Los prejuicios producen discriminación y, en mi caso, el niño nunca tuvo la posibilidad de desarrollar sus capacidades y menos aún su vida emotiva. Cuando un sistema de turno dirige el crecimiento de un niño, el niño tendrá ...cuarenta ...cincuenta ...padres y madres.

Antes de escribir más sobre mi libro quisiera decir algunas palabras sobre mi vida profesional y cuál es el motivo por el que he escrito este libro.

Soy jurista. Recibí mi licenciatura en Derecho en 1972. Por eso, llevo trabajando como jurista más de treinta años. Once años trabajé como burócrata para el Gobierno. En 1984, recibí un cargo como Ombudsman -defensora de los pacientes-. Fui la primera defensora de los pacientes en Noruega. Trabajé como defensora de los pacientes durante cuatro años. Después comencé a trabajar como Profesora en la Escuela 
Superior de Bodo. Bodo está en el Norte de Noruega. También he trabajado como abogada.

En 1997 un enfermero me pidió defenderlo. Este enfermero es extranjero, había trabajado con el niño por un año y medio. Durante este tiempo el niño tuvo una integración fantástica. Este enfermero trataba al niño de una manera diferente a la mayoría en el hospital.

Para este enfermero el niño, en primer lugar, era un ser humano, en segundo lugar, era un paciente. En realidad, el niño crecía saliendo del papel de paciente para ir hacia el papel de un niño.

Este enfermero tenía el fuerte deseo de que el niño tuviera una posibilidad de crecer en una familia normal.

Los pensamientos y las acciones de este enfermero eran un desorden, una rebelión frente a la cultura del hospital. Por eso, apareció un gran conflicto: “¿Era el niño un paciente que sólo necesitaba cuidado y oxígeno o era el niño un ser humano que necesitaba crecer y desarrollarse?"

El resultado del conflicto profundo fue que este enfermero fue echado y denunciado a la policía. Algunos profesionales del hospital usaron medios deshonestos para acusarle y juzgarle. Fue acusado de atentar contra la integridad física del paciente. Hubo tres causas criminales y penales antes de que, finalmente, fuera absuelto.

Yo fui su defensora.

La última causa fue en julio de 1999. En ese momento, el niño tenía diez años. Durante la tercera causa, el juzgado nombró un médico para aconsejar al juzgado. Este médico es experto en enfermedades de pulmón y especialmente en niños que tienen problemas con sus pulmones. Este médico dio a mi enfermero su apoyo completo.

"Que los niños no crezcan en un hospital" dijo él.

Este médico también reveló que el niño había recibido un tratamiento erróneo. El niño había recibido menos entrenamiento físico del necesario. Pero lo peor era que el niño llevaba una cánula dentro de su cuello. Este médico opinaba que esta cánula no era necesaria para su tratamiento. Esta cánula había ocasionado al niño muchos problemas: para hablar, para comer, problemas infecciosos (un objeto, una cosa desconocida dentro de su cuerpo).

Durante las tres causas, y porque luché durante largo tiempo para conseguir una familia para el niño, aprendí mucho sobre discapacidades y sobre los prejuicios frente a las discapacidades. 
Desde mi punto de vista es muy grave cuando los médicos, enfermeros y trabajadores sociales tienen prejuicios frente a las discapacidades. He escrito esta historia con la esperanza de poder concienciar a las personas que trabajan con niños que son discapacitados, contarles a ellos que los niños, en primer lugar, son seres humanos y, después, en segundo lugar, son pacientes. Además, hacer conscientes a los trabajadores (profesionales) de que cada uno tiene la responsabilidad de reaccionar cuando se ve un error en el tratamiento, tanto físico como psíquico.

En una institución hospitalaria hay una imposibilidad para que un niño reciba satisfactoriamente sus fundamentales necesidades de amor y cuidado. Desde mi punto de vista, el amor es lo más importante para todos los niños. Por eso, los niños crecen en las familias y no en instituciones. El cuidado que se recibe en un hospital es diferente del cuidado que se recibe en una familia. En mi caso, los profesionales en el hospital, nunca vieron esto, o para ellos la vida emotiva no era importante.

Un niño con discapacidad puede ser vulnerable. En esta situación es muy importante tener padres que vean al niño y le comprendan. También es importante tener padres que luchen por los derechos del niño.

En la realidad, vivimos en un mundo que discrimina a los discapacitados.

La pregunta de mi libro es:

$¿$ Tiene un niño con discapacidad derecho a crecer en una familia?

$¿$ Tiene un niño con discapacidad derecho a exigir algo de sus padres?

En mi libro, yo digo: "en Noruega No".

Ninguna autoridad pública estaba en condiciones de contestarme.

Mi libro contiene veinte capítulos. Yo pruebo acompañar al niño desde su nacimiento hasta que el niño tenía once años y ocho meses y se mudó al municipio de sus padres. El niño recibió una vivienda propia cerca de la casa de su madre. Yo también escribo sobre las tres causas concernientes al enfermero extranjero y mi lucha para conseguir una familia al niño.

Aunque mi historia es una historia real, yo no conozco la historia completa. La mayoría de mis conocimientos los he recibido durante las tres causas.

El niño era un paciente, por eso los profesionales estaban obligados a mantener el secreto profesional. 
Yo he sido una extraña que ha hecho una lucha por el niño. Mi opinión primordial sobre lo que necesita un niño con discapacidad ha sido diferente a la de los responsables en el hospital y la de los padres del niño. En realidad yo he hecho una lucha frente al hospital y a los padres. En esta situación para mí no ha sido deseable y tampoco posible entrevistarlos. Yo supongo que ni el hospital ni los padres querrían que mi libro se publicara.

La historia del niño va a ser silenciosa.

Es fácil decir la historia sobre el niño únicamente. La unicidad sobre la historia es que la historia fue publicada a raíz de las tres causas penales.

En este artículo yo pruebo dar una descripción corta del contenido de mi libro.

\section{LOS PRIMEROS AÑOS DEL NIÑO}

El niño nació en marzo de 1989 en un hospital local en el Norte de Noruega. El niño es gemelo. Durante el nacimiento aparecen complicaciones y el niño recibe líquido amniótico dentro de los pulmones, en esa situación se aplica oxígeno. El pulmón derecho es lesionado, por lo que se compacta. El niño tiene problemas para respirar y después de cuatro o cinco horas es transportado al hospital de Nordland en un avión ambulancia. Un día más tarde el niño es enviado al hospital del Estado de Oslo. Durante la estancia allí se constata que el niño tiene un problema congénito de pulmones subdesarrollados.

En el hospital del Estado el niño recibe una traqueotomía (se le hace un agujero en el cuello dentro de la tráquea y allí se coloca una cánula). Durante su estancia en el hospital el niño tiene todo el tiempo un pulmón artificial. El niño está tres meses en el Hospital del Estado. Tiene una situación dramática en su vida. Hábiles profesionales de salud y equipos avanzados de médicos-técnicos salvan su vida.

El niño vuelve a la sección de prematuros en el Hospital de Nordland en junio de 1989. El niño está acostado y con un respirador durante la Navidad. La sección de prematuros está abarrotada y los profesionales tienen que hacer mucho..., demasiado. El niño está acostado en un rincón.

Durante este período ya vemos los contornos de lo que será la vida del niño. El niño no tiene a nadie en el mundo. Los padres se mudan separados y tienen sus propios problemas. Además, los padres viven 
lejos del hospital de Nordland. No obstante, los padres lo visitan los fines de semana.

El niño tiene su primer año. En este período, lo más importante para un niño es que establezca una relación primaria, una madre, un padre u otro contacto íntimo, no estar allí todo el tiempo solo.

En la primavera de 1990 el niño se muda a la sección intensiva. Los pulmones comienzan a funcionar mejor. Los profesionales comienzan gradualmente a deshabituar al niño del respirador. En este momento, los profesionales están de acuerdo sobre que el niño necesita una familia propia. El pensamiento de una familia provisional también se esta discutiendo, pero se queda en el pensamiento.

En lugar de conseguir una familia provisional, el niño se queda como paciente. El hospital de Nordland quiere que el niño regrese al hospital local donde el niño nació. El objetivo es que el niño llegue más cerca de su familia biológica. Este es el primer plan, después hay muchos planes y todos exigen tiempo y dinero para ser llevados a cabo.

A principios de noviembre de 1990, el niño se muda al hospital local. Antes del año Nuevo el niño ya ha vuelto dos veces al hospital de Nordland. El problema es que no hay médico anestesista en el hospital local.

Durante el invierno de 1991, el niño cae más enfermo y está en el hospital de Nordland durante dos meses. Los profesionales consideran reducir el tratamiento. Pero el niño tiene una increíble fuerza vital y sobrevive.

En el verano de 1991, el niño está durante largos periodos en su hospital local.

El dieciseis de septiembre de 1991 fue en realidad un día fatal para el niño. Aquel día el niño vuelve al hospital para siempre. A partir de ese momento el hospital se hace cargo del niño completamente. El hospital establece un equipo alrededor del niño. El equipo consiste en doce enfermeros y asistentes, técnicos sanitarios (ATS) y muchas guardias suplementarias. El niño se muda con el equipo a dos habitaciones en la sección intensiva. Para el hospital es un proyecto interesante, y este proyecto tiene su presupuesto propio. Progresivamente, el presupuesto suma quinientos mil euros por año. Quinientos mil euros para cuidar de un niño durante un año.

En la primavera de 1992, la situación es más estable. El niño usualmente duerme toda la noche y es más fácil manejar su discapacidad. En esta situación, es fácil pensar que la madre o el padre vean la posibili- 
dad de llevarse el niño a su casa. Sucede lo contrario: ni la madre ni el padre lo visitan más semanalmente. Especialmente a la madre le cuesta más retornar. Al niño le falta el desarrollo de una relación íntima.

El equipo se ha establecido y comienza su vida propia. Desde mi punto de vista el equipo trata al niño como a un paciente y no como a un niño que tiene una discapacidad. El niño es pasivizado en lugar de ser activado, estimulado.

El equipo es complementado por una pedagoga especial. Todo el equipo va a aprender a hablar por signos. Este es un idioma que usan los sordos. El niño no es sordo. El niño comprende todo lo que se habla. El niño tiene problemas con el habla a causa de la cánula dentro de su cuello. El hospital cree que la cánula es necesaria para tratar al niño con respirador.

El equipo ha recibido la instrucción y la conciencia de que el niño es un paciente único y que no hay otro en Noruega con una enfermedad similar. Por supuesto que hay muchos niños con pulmones que están lesionados. Pero ellos no crecen en el hospital, y los niños usan máscaras nasales y respirador. Pero el niño no tiene a nadie que luche por él e investigue qué usan otros niños que están en situaciones similares. De todos modos la enseñanza con "señas para hablar" es una posibilidad de comunicarse con el niño.

Cada día, dos personas del equipo toman un paseo con él fuera del hospital. El niño está sentado en su silla de ruedas.

Fuera de su habitación, el niño encuentra a mucha gente, en el pasillo del hospital. Los trabajadores del hospital lo conocen y muchas personas quieren saludarlo. El niño es como una mascota en el hospital.

Entonces suponemos los contornos de lo que sería su vida en adelante.

El niño está solo y el niño tiene una serie de relaciones casuales. Todos quieren ser amables con él, pero las relaciones son superficiales.

Ninguna relación es profunda y de carácter permanente.

La Nochebuena es la más importante noche para la familia en Noruega. Usualmente las familias están todas juntas esta noche. El niño siempre pasaba la Nochebuena solo, con las personas que casualmente estaban de guardia.

La primavera de 1993 un pensamiento está madurando: crecer en una sección intensiva no es satisfactorio para un niño. No obstante el pensamiento médico-técnico es muy dominante. 
El equipo electromecánico que el niño necesita está en muchas casas y es fácil para una madre o un padre aprender a usarlo.

Esa primavera queda claro que ni su madre ni su padre están en condiciones de cuidarle. La madre no quiere que el niño crezca en una familia provisional. En esta situación es fácil para el hospital continuar a cargo del niño. Es impensable para el hospital tomar un conflicto frente a la madre. Por eso el hospital nunca toma contacto con la Tutela de niños para contar que en el hospital hay un niño que necesita una familia.

Los profesionales en el hospital opinan que sería mejor para el niño crecer como un paciente en el hospital que crecer en una familia provisional.

En la primavera de 1993, el hospital determina que el niño se mudará a un piso propio con el equipo. El hospital hace un proyecto para llevar a cabo esta mudanza. Mucha gente está dedicada a este proyecto. Nadie piensa en la vida emotiva del niño.

En este tiempo, Noruega tenía mucho conocimiento sobre discapacidades, especialmente sobre daños después de estancias largas en instituciones (ONU "artículos estándar para iguales posibilidades para personas con discapacidades, Goffman Erving, Institución y ser humano, análisis del Estado Noruego sobre condiciones de vida de las personas con discapacidad intelectual).

El problema para el hospital es que el hospital piensa en el niño como un paciente. En esta situación no es importante que el niño reciba satisfactoriamente sus fundamentales necesidades de amor y cuidado. El hospital se toma dos años en el proyecto. En enero de 1995 el niño se muda a su piso propio con el equipo.

\section{LA INTEGRACIÓN. EL PERÍODO CON BERTHOLD}

Durante 1995 suceden muchos cambios en la vida del niño. El niño se muda del hospital a su piso propio y un enfermero extranjero recientemente ha comenzado en el equipo ( el enfermero se llama Berthold). $\mathrm{El}$ apartamento tiene dos pisos. En la planta baja hay un salón y la cocina. Fuera del salón hay una terraza. Además, hay un anexo para sus tanques de oxígeno. En el primer piso, hay tres dormitorios y un baño. El niño recibe su dormitorio propio. Un dormitorio es usado como oficina. El tercer dormitorio es para la madre o el padre, quienes pueden usarlo cuando ellos le visitan. El piso está amueblado como una casa normal. 
Aunque hay un equipo técnico y a pesar de su discapacidad, el piso está en una urbanización normal. El hospital ha hecho un esfuerzo fantástico para crear una casa buena para el niño. Pero lo que falta en comparación con una casa normal es que no hay una madre o un padre. Aunque hay un dormitorio para los padres, no es usual que ellos lo utilicen, normalmente hacen noche en otros lugares cuando viajan para visitarlo.

A pesar de la mudanza el niño hace grandes progresos. El niño necesita menos oxígeno y le viene un tiempo con más actividades.

Berthold comienza en el equipo en el otoño de 1994. En ese tiempo, Berthold tiene cuarenta y tres años y trece de experiencia como enfermero. La jefa del equipo toma una licencia desde noviembre de 1994 hasta septiembre de 1995. En este periodo, Berthold es el jefe del equipo.

Rápidamente, Berthold descubre que el niño ha sido pasivizado completamente. Los miembros del equipo habían hecho todo para él. El objetivo para Berthold es que el niño consiga vivir tan normal como sea posible a pesar de su discapacidad.

Cuando Berthold comienza en el equipo, el niño recibe comida por sonda, y que la bebe por boca. Es líquido dentro de paquetes que se toman de la nevera. En el líquido hay todos los alimentos necesarios. La comida es un tanto monótona porque le falta gusto.

Berthold comienza con sopa de tomate. Después él prueba más, con un poco de nata, un poco de mantequilla y después sopa de coliflor.

Al principio, Berthold tiene problemas con algunos de los miembros del equipo. Ellos opinan que la sopa sabe mal y que no están seguros de cuántas calorías, minerales..., hay dentro de la sopa. Para ellos, es importante que un especialista en dietética apruebe la sopa.

En este tiempo, Berthold ha sido el jefe del equipo y por eso él tenía la posibilidad de dar al niño comida normal. Un día están en la cocina con una merluza para hacer el almuerzo. El niño lleva un sombrero de cocinero y asiste con la cocina.

Berthold está preocupado porque el niño no habla. El problema es la cánula dentro del cuello. En el equipo se habla sobre un "hablar cánula". Berthold encuentra esta "hablar cánula" dejada en un armario. Berthold sabe cómo se monta el equipo y lo hace. Algunos días después el niño es operado y le ponen "el hablar cánula". Entonces el niño puede decir sus primeras palabras. 
Pero hay problemas para continuar el desarrollo. La estimulación monótona que el niño había recibido durante muchos años había hecho algo con su personalidad. Además, muchos miembros del equipo continúan hablando por él. Claro que es más fácil hablar por él que esforzarse, que lograr que el niño pueda expresarse por sí mismo. Esto exige paciencia. Además, la mayoría del equipo continúa usando "señas para hablar" que ya he nombrado precedentemente bajo el punto II. Los primeros años del niño, cuatro años después, durante la tercera causa un médico revelará que la cánula no era necesaria para su tratamiento.

El niño era silencioso no a causa de su discapacidad sino a causa del tratamiento erróneo.

Hasta que Berthold comienza en el equipo el niño no puede ir a algunos lugares sin ayuda. Ni sube la cama, ni se levanta de la cama. No camina del dormitorio al salón. No sube las escaleras.

Gradualmente, el niño aprende a montar en bicicleta (la bicicleta tiene tres ruedas), y también aprende a caminar. Durante este tiempo el niño desarrolla fuerza y confianza, y el niño está muy orgulloso y feliz de sus progresos.

\section{Una historia de ese tiempo:}

"Es verano de 1995. El tiempo es bueno. La guardería infantil tiene un día en el campo con bosque y montaña.

Berthorld y otra persona del equipo están junto al niño. Este día el niño solamente usa un carrito de apoyo. El niño pasea por sí mismo alrededor, en la hierba. De repente el niño va a una cuesta y entonces el carrito toma velocidad.

Todo el niño es como una gran pregunta y se ve que el niño no consigue determinarse: va a llorar o va a reir.

El carrito viaja con rapidez salvaje cuesta abajo, y el niño prácticamente cuelga de ella, sus pies dan patadas. La cuesta es corta y el niño consigue mantener el equilibrio y sale bien librado. Los espectadores le aplauden, esto le da ganas de más y ahora tiene el "ojo avizor" para tener una aventura nueva.

El niño está de pie y atisba entre los arbustos que hay dos o tres chicos que hacen cabriolas. Primero el niño señala a los chicos y después a su cabeza: "tontos". Berthold dice que en esta situación hay solamente una cosa para hacer: levanta al niño arriba, entre los arbustos. De nuevo Berthold especula sobre cómo el niño lo consigue. Pero el niño rápidamente está más ocupado con empellar a los otros chicos." 
Los padres del niño viven en una isla grande a las afueras de Bodo (el hospital está en la ciudad de Bodo). El niño, en este tiempo, nunca había estado ni en la casa de su madre, ni en la casa de su padre. Berthold opina que es importante conocer sus raíces.

El hospital a veces había pensado que el niño tiene que tener la posibilidad de visitar las casas de sus padres. Pero el hospital siempre está preocupado por la salud del niño. Berthold, en cambio, opina que la hiperprotección del niño también es dañosa para su salud y desarrollo.

Berthold piensa y piensa. Berthold sabe que al niño le gustaría ser llevado y también le gusta recorrer. Berthold tiene un plan: alquilamos un coche y tomamos el transbordador a Lofoten, donde viven los padres. Berthold me ha dado esta descripción sobre el viaje:

"Una mañana bonita de viernes, tomamos el equipo del niño dentro del coche. El niño, tres miembros del equipo y yo nos condujimos al muelle para tomar el transbordador. El tiempo era bueno y satisfechos en el grupo entramos en el barco. Fue maravilloso para el niño investigar el barco y subir las escaleras hacia la cubierta con sol. Después de una siesta el niño pudo vislumbrar las islas de Lofoten en el mar. El niño estaba entusiasmado para bajar al coche.

Llegamos a la casa de la madre y el niño estaba bien. Tuvimos una tarde agradable. Por la noche el niño junto a dos miembros del equipo estuvieron en el hospital local, aunque no era necesario.

El sábado por la mañana las tres mujeres del equipo estuvieron andando por las tiendas de la ciudad. El niño y yo fuimos con el barco de la madre. Al principio, la madre llevó la rueda del timón, un poco más tarde el niño tomó posesión como capitán. Esto que pasó tenía un efecto mucho mayor que sus experiencias y habilidades con su bicicleta, no hizo falta usar salvavidas.

Después del viaje en barco visitamos a un tío y a una tía del niño. Allí hubo café y pasteles de crema, el niño se sentía muy a gusto con el pastel de crema. Seguidamente un nuevo paseo en coche hasta que caímos en manos de los abuelos. Los abuelos paternos viven en unos alrededores idílicos. Otra vez el niño estaba consentido. Se sintió "el jefe", disfrutó todo el fin de semana de ser el centro.

Durante el viaje en transbordador a casa, el niño entretuvo a los otros en el barco. Él con su gorra roja del revés, delante de la frente, saltó un "jo jo" en toda la cafetería y les encantó a todos. 
Entonces llegamos a casa a las 22 horas. El niño estaba en mejor forma que los otros". Berthold me ha contado que él nunca ha visto al niño tan contento como este fin de semana.

Después de la llegada, las tres personas que habían estado en Lofoten exigieron más dinero que el acordado. Hubo una acción de reclamo y muchos problemas. Muchos años pasaron antes de que el niño visitara otra vez a sus padres.

Berthold vive en una casa junto al mar. Tiene un barco de vela. Berthold muchas veces lleva al niño a su casa, y cada vez el niño quiere visitar el barco de vela. Berthold tiene revistas de barcos que le da al niño para que mire.

El niño tiene evidentemente oído musical. Berthold toma mucha música en un cassette, que también le da, entonces el niño puede estar sentado un buen rato ojeando una revista de barcos y al mismo tiempo escuchando música, que el niño denomina "barcomúsica".

La guardería infantil incluye niños con diferentes dificultades. Es una oferta adecuada para promover el desarrollo de niños con discapacidades. Los niños con discapacidades usualmente son aceptados por otros niños, y no son de ninguna manera susceptibles a bromear y molestar a los niños sin discapacidad (análisis noruego público, noviembre de 1985: 34. Lars Grue. Familias acostumbradas a niños distintos. Oslo: Ad. Notam Gyldendal 1993).

Muy desde la base para ser un ser humano social con habilidad para relacionarse con otros y establecer contactos por sonido durante los años de la niñez. Para el niño, que tiene una situación en la vida tan especial es muy importante estar en la guardería. Después de que Berthold comenzó en el equipo el niño viene más regularmente a la guardería.

\section{EN EL OTOÑO DE 1995, EL NIÑO TIENE SEIS AÑOS Y ME- $\mathrm{DIO}$}

Este año, el niño muestra un desarrollo fantástico. El niño se ha levantado de la cama, él ha comenzado a caminar y a montar en bicicleta. El niño ha comenzado a comer comida normal y él, gradualmente, ha aprendido a vestirse. El niño no usa más pañales y se lava por sí mismo. El niño ha mostrado también que le gustan los retos. Durante 1995, el niño nunca estuvo en una situación aguda. Cuando el niño recibe su oxígeno él funciona igual que otros niños.

¿Por qué el niño continua siendo un paciente? 
Cuando es paciente en el hospital es usual que un médico lo visite todos los días (visita médica). Una pregunta central durante la visita médica es cuándo se le dará el alta.

En un hospital lo usual es estar ingresado temporalmente. Un hospital tiene como objetivo curar al paciente y lo más pronto posible darle de alta. En la situación del niño lo contrario sucede. Cuando el niño con el equipo se mudan a su vivienda están en realidad abandonándolo a sí mismo.

Durante 1995 la enfermera jefe solamente les visita una vez, en el cumpleaños del niño. Los médicos responsables no le visitan regularmente, por termino medio cada dos meses. La madre también visita al niño irregularmente. En este tiempo, puede, en efecto, pasar tres meses sin que la madre lo visite.

Las autoridades sanitarias (que ejercen el control) le visitan una vez. Una vez por semana el niño va al hospital para un reconocimiento médico. Esto es sólo de carácter rutinario para la medicación.

El niño está en una situación extraña. Los médicos tienen la responsabilidad para dar el alta, pero no están preocupados por este asunto. El niño está ubicado en su piso y entonces los médicos pueden concentrarse en la medicación.

Los enfermeros y los asistentes sanitarios cuidan con cariño. Ellos tienen la responsabilidad para cuidar un paciente y lo hacen hasta que el médico determine dar el alta. No tienen ninguna tradición ni cultura para cuestionarse si es justo o si hay derecho a que un paciente esté siempre internado en el hospital. Los enfermeros históricamente pertenecen a una cultura sumisa (Åshild Sletteba 1997, Asistencia médica y ética. Oslo: Ad. Notam Gyldendal). Son los médicos los que diagnostican.

Cuando el equipo está abandonado a sí mismo es evidente que los profesionales asimilan muchos conocimientos sobre el niño, su crecimiento, desarrollo y su relación con los padres. El alcance y la profundidad de estos conocimientos son imposibles de asimilar para los médicos y la jefa de enfermeros, ya que ellos están en el hospital.

Desde mi punto de vista, lo ideal sería que el equipo todo junto se presentara al jefe médico y a la jefa de enfermeros y les contaran sus experiencias. El equipo debería reconocer que un equipo de hospital no es una madre o un padre para el niño. Lo presupone una conciencia ética y profesional sobre el ejercicio de la profesión que en este caso parece ausente entre la mayoría de los involucrados. 
Se puede preguntar hasta qué punto sus conductas son representativas y universales cuando éstas afectan la actitud hacia discapacidades generales y especialmente a los niños con discapacidades.

\section{VIGILANCIA E INSPECCIÓN}

La primavera de 1995, vienen al piso del niño una representante (enfermera) de las autoridades sanitarias y una representante ( trabajadora social) del Gobierno Civil. Ambas vienen en el ejercicio de su cargo para vigilar que el niño esté bien y que los trabajadores hagan sus trabajos. Inmediatamente, las vigilantes reciben una buena impresión. El niño vive en un piso bonito y en todo lugar hay limpieza. El piso está amueblado como una casa normal y hay mucha gente para cuidarle. Los trabajadores tienen un buen tiempo y les pueden servir café y pasteles.

Lo triste es que las vigilantes no hacen cuestiones sobre la necesidad de que el niño va a crecer solo en una institución y crecer con un grupo que responde a un sistema de turnos. Eso es porque las vigilantes no opinan mal. Cuando se es un niño con discapacidad no se puede exigir que los padres lo cuiden.

Las vigilantes no consiguen ver que un niño con discapacidad tiene la misma vida emotiva que un niño sin discapacidad. Las vigilantes tampoco consiguen ver que hay una alternativa a la institución: un hogar provisional.

Después de la inspección, las Autoridades Sanitarias y el Gobierno Civil están de acuerdo sobre que solamente las autoridades sanitarias van a seguir la vigilancia. La causa es que el niño es un paciente y está sometido a la sección niños del hospital.

Muchos años pasarán antes de que la vigilancia visite al niño. La única nueva visita tiene lugar en diciembre de 1997. La razón de esta visita es que yo he iniciado una lucha para conseguir una familia para el niño. En el informe de esta visita las autoridades sanitarias destacan los esfuerzos fantásticos que ha hecho el hospital por el niño.

\section{EL CONFLICTO TRABAJADOR}

En el verano de 1995, aparece gradualmente un conflicto dentro del equipo: ¿es el niño un paciente que sólo necesita cuidado y oxígeno o es el niño un ser humano que necesita crecer y desarrollarse? 
En el otoño de 1995, el equipo se divide de alguna manera en tres grupos. Un grupo deja el equipo. Estas personas apoyan a Berthold y ven que el niño necesita una familia. Se sienten impotentes. Esperan que alguien con poder en el sistema hospitalario reaccione. Las personas no toman por sí mismas contacto con los responsables para conseguir un cambio en la situación del niño. Se comportan pasivamente. $\mathrm{La}$ mayoría del grupo, como se queda, nunca comprende que es negativo para el niño crecer en un equipo de hospital, más bien al contrario. Para ellos es un proyecto interesante y ellos están orgullosos de trabajar con un paciente que tiene una enfermedad única. Berthold, por su lado, está gradualmente más o menos solo con sus actividades y su sueño sobre que el niño crezca en una familia.

El conflicto florece completamente cuando la jefa del equipo vuelve de su licencia. Esta señora ha sido la jefa desde que el equipo fue establecido. Muy desde la base caracterizada por la hiperprotección y la pasividad que fue sentada durante esos años en que ella guiaba el equipo.

El grupo se une frente a Berthold, entonces calumnias y falsedades comienzan a florecer. Berthold entre otras cosas es acusado de tomar duramente del brazo al niño y quitarle el oxígeno como pena. El resultado del conflicto es que Berthold es suspendido y denunciado a la policía.

El veintinueve de enero de 1996, es el último día de Berthold en el equipo.

\section{LA PRIMAVERA DE 1996. EL NIÑO TIENE SIETE AÑOS}

Durante la primavera de 1996, el niño tiene siete años. Él todavía no ha conseguido dormir ni una noche en una casa normal. Las posibilidades del niño para conseguir una familia se eclipsan frente a la causa penal contra Berthold.

¿Qué comprende el niño sobre lo que pasa? El niño ve que Berthold de repente desaparece. Berthold no tiene el permiso para tomar contacto con él y decirle adiós.

Para el niño hay un vacío al que nadie puede darle una explicación razonable. 


\section{LA CAUSA CRIMINAL Y PENAL}

Desde el momento en que la dirección del hospital denunció a Berthold a la policía, una pequeña bola de nieve comienza a rodar. Poco a poco, la bola de nieve rueda con acelerada velocidad y se convierte cada vez más y más grande. Nadie consigue, de alguna manera, detener la bola de nieve. Poco a poco, se va descubriendo una realidad que nadie consigue reconocer como errónea. El objetivo es conseguir que Berthold sea juzgado y quede fuera del hospital y esto es lo que va a acaecer sin que se tengan algunos pensamientos sobre si es justo $o$ injusto. En realidad el niño está metido en el remolino. En tanto que se tiene una cabeza de turco en el sistema, se está más ocupado en conseguir echarle, en lugar de que el niño pueda crecer en una familia. El sistema del hospital usa mucho tiempo y muchas fuerzas para conseguir que Berthold sea juzgado y esté fuera del hospital -pienso cómo sería si el sistema hubiera usado tantas fuerzas y tantos pensamientos para conseguir una familia para el niño-.

Inmediatamente después de la denuncia, uno de los periódicos locales recibe conocimientos sobre la causa. El resultado es un artículo con un titular gigante y una imagen de Berthold: "Enfermero denunciado por abuso contra un niño que necesita tratamiento intensivo". Dentro del artículo se exagera sobe el hecho de que Berthold ha negado al niño oxígeno para penarle. Otros periódicos también escriben sobre la causa.

El grupo que se queda se convierte todavía en más unido. El veintiseis de marzo de 1996, la jefa de enfermeros de la sección niños y la jefa del equipo son interrogadas por la policía. Después de los interrogatorios el equipo tiene una reunión. La jefa de enfermeros en la sección niños también participa. Durante la reunión el equipo decide que los "informes diarios" se van a inutilizar. En estos informes se anotan informaciones detalladas diarias sobre el niño durante el período en el que Berthold trabajó ahí, los informes decían podía estar sin el suministro de oxígeno durante más horas. Esto pasaba independientemente de que Berthold estuviera en el trabajo o no. La causa era que el desarrollo de los pulmones del niño había avanzado tanto más, que el niño durante periodos, en los cuales su estado de salud era bueno no necesitaba el suministro del oxígeno todo el tiempo. En el mencionado artículo del periódico aparece que Berthold usa la falta de oxígeno para penarlo. Por supuesto, Berthold no ha usado el oxígeno para penarlo. Inutilizando "los informes diarios" las circunstancias y el hecho sobre la menor necesidad del niño en el suministro de oxígeno se encubre. Esto se hace en un acta escrita a mano. 
En el primer contacto con la causa criminal y penal que ha sido reanudada, Berthold y yo llegamos a saberlo. Nadie en el grupo cuenta ni a la policía ni al juzgado sobre lo que pasa en la reunión.

El grupo ha hecho una norma para todos los que van a ser interrogados. Está permitido "engañar" para que Berthold sea acusado, juzgado y quede fuera del hospital.

La presión del grupo tiene que haber sido enorme. Cada miembro individual debe haberse sentido sofocado, en forma individual los miembros deben haber pensado que el niño es más que un paciente, que necesita mucho más que oxígeno y cuidados, que un hospital es un lugar totalmente inadecuado para que un niño crezca, pero estos son pensamientos que no se aceptan.

En la pared dentro del piso cuelga la instrucción del médico anestesista,sobre el uso del pulmón artificial y el suministro de oxígeno. En esta instrucción no está escrito que el niño vaya a tener constantemente suministro de oxígeno. Se hace una instrucción nueva donde está escrito que el niño necesita constantemente suministro de oxígeno.

Tanto la policía como el juzgado creen que la instrucción nueva estaba en vigor cuando Berthold trabajaba en el equipo.

La policía lleva un tiempo largo sobre la causa. La policía se confía en el grupo. Para la policía es impensable que los trabajadores de la salud pública no cuenten a la policía la verdad.

El doce de marzo de 1997, Berthold se halla bajo acusación de atentar contra la integridad física del niño.

\section{MI ENTRADA EN LA CAUSA}

Entonces, Berthold se halla bajo acusación, está desesperado. Su abogado en ese tiempo es pasivo y Berthold quiere un nuevo abogado. Berthold toma contacto conmigo. Le digo que no soy experta en derecho penal, pero que sí tengo muchos conocimientos sobre la vida hospitalaria.

Mi primera reunión con Berthold es el dos de abril de 1997. Después de nuestra primera conversación, le digo: "Berthold, tengo dos personas que se salvarán en esta causa".

Entro en contacto con la jefa del hospital. La jefa es enfermera y tiene niños propios. Yo soy clara en mi explicación. Yo digo en cualquier caso que el hospital usa del dinero y recursos, pero nunca eso será suficiente. Un niño tiene derecho a crecer en una familia. Un niño no tiene 
cubiertas sus necesidades más fundamentales en el hospital. La jefa del hospital muchas veces me dijo que ella comprende lo que yo opino. Lo que ella no comprende es que ella como la jefa del hospital tiene que poner orden en la causa. Yo le digo que es criminal no anunciar a la Tutela de niños. La conversación termina en que la jefa tomará contacto con la sección niños y organizará una reunión para mí.

Tengo una reunión con muchos profesionales de la sección niños. Los profesionales no me comprenden cuando hablo sobre necesidades fundamentales de un niño. Después de que estoy trabajando con la causa durante un par de semanas, envío una carta larga a la Fiscalía del Ministerio Público. Le solicito a la Fiscalía retirar la acusación e investigar el problema principal: el niño crece en un hospital.

Hago referencia a disposiciones legales pertinentes de nuestra Ley Médica y de la Ley de Tutela de niños. Pero la Fiscalía ya había tomado una resolución y, por eso, hay una imposibilidad de retirar la acusación. Investigar el problema principal es demasiado complicado. Por supuesto, es más fácil acometer contra una persona que contra un sistema.

La Fiscalía consigue apoyo en la primera causa y Berthold es juzgado. Esto es el verano de 1997.

\section{MI PRIMERA LUCHA POR EL NIÑO}

En el verano de 1997, inicio en serio la lucha para que el niño consiga una vida mejor. Durante la causa criminal y penal que ha durado una semana, se ha llegado a comprender cómo es para el niño crecer en un hospital. No es una experiencia buena. Esto no sólo es a causa de las palabras que son dichas dentro del banquillo de los testigos, sino también por las actitudes de los profesionales en el grupo (que se queda) revelan.

Lo primero que hago es tomar contacto con una oficina pública provincial. Esta oficina tiene como objetivo buscar familias adecuadas para los niños que necesitan una familia provisional. Esta oficina también trabaja para buscar familias adecuadas para niños con discapacidades. Ese verano, tengo muchas conversaciones con la jefa de la oficina. Ella me comprende completamente. Pero la oficina no puede hacer nada antes de que la oficina haya recibido una solicitud de la Tutela de niños. Aquí se ven los contornos de lo que hace que el niño nunca consiga crecer en una familia. La jefa ve que al niño le falta algo fundamental en su vida, pero aún así no puede salir de su papel formal como empleada pública. Ella no puede en virtud de sí misma, como prójimo, simplemente, tomar contacto con la sección niños del hospital y contarles lo 
dañoso que es para un niño crecer en un hospital. Cuando esa formalidad no está en orden, los profesionales no pueden abordar un asunto, aunque ellos vean lo malo que es esto para un niño. Lo correcto formalmente, de acuerdo a la ley de Tutela de niños, es que el organismo de Tutela Municipal tiene que dar curso a un asunto en la junta protectora de menores. Cuando se ha dado curso a un asunto, se dirige a la oficina pública nombrada para ayudar a conseguir un hogar provisional.

El diecisiete de agosto de 1997, envío una carta larga a la Tutela de Niños Municipal. Envío copia de mi carta a los siguientes destinatarios: el jefe del Hospital, Ministerio de Salud Pública, autoridades provinciales, al Gobierno Civil y a la Policía. También envío una copia a la madre del niño. Por supuesto, mi carta es una llamada de socorro para que el niño consiga una vida más digna. No viene ninguna reacción de ellos a quienes les he enviado una copia.

La Tutela de Niños Municipal me escribe que no hay ninguna razón para investigar la causa. La argumentación es que el niño es un paciente. Además la Tutela de niños se refiere a las Autoridades Sanitarias Provinciales que tienen vigilancia y que ellos eventualmente tienen que tomar la iniciativa de cambiar la vida del niño.

Es fácil ver que la Tutela de niños toma el camino más rápido. El niño ha recibido la clasificación de paciente y en tanto que él tenga esta clasificación, la Tutela de niños no necesita molestarle.

Desde mi punto de vista hay algo más profundo tras la resolución. No es un fenómeno nuevo que a los niños con discapacidades, sus padres ni quieren o ni pueden cuidarlos, son "apiñados" lejos, dentro de intituciones con sostén y aprobación del Ministerio Público. Desde que el danés Niels Erik Bank-Mikkelsen en 1959 inició su trabajo para que los discapacitados vayan a conseguir vidas más dignas y normales (Kristjana Kristiansen, Normalización y Estimado del papel social. Oslo. Kommuneforlaget, 1994) y hasta hoy ha pasado un desarrollo enorme. Esto ha conducido a que tales intituciones hayan sido clausuradas. La cuestión es que, sin embargo, dentro del pensamiento de la gente, también de los profesionales, hay algo que remuerde en las conciencias. ¿Nosotros rechazamos lo que es discapacidad o distinto sin que nosotros mismos seamos conscientes? (Foucault Michel. La historia de la locura, Oslo Gyldendahl, 1973).

Tendemos sin duda más fácilmente a identificarnos con los padres que tienen un niño con discapacidad que con el niño discapacitado. Muchos de los niños que estaban en las instituciones nunca han recibido la visita de sus padres. Alguien a quien ellos simplemente han dado por perdido. Los padres nunca les han preguntado nada a sus niños. A 
pesar de esto, se está ocupado en la lástima por los padres (Análisis del Estado Noruego sobre condiciones de vida de las personas con discapacidad intelectual).

La Tutela de niños tiene como tarea asegurar que los niños que viven en circunstancias que puedan dañar su salud y desarrollo, reciban ayuda necesaria y cuidado. La Tutela de niños va a contribuir a que los niños y jóvenes reciban crecimiento seguro. Por esto, los pedagogos de la tutela de niños y trabajadores sociales tienen educaciones sólidas y, por supuesto, experiencia práctica.

¿Qué pasa cuando la Tutela de niños en una causa como ésta no logra ver al niño y sus necesidades? En realidad hablamos sobre prejuicios. Los prejuicios surgen entre, otras cosas, por la falta de conocimiento, de contacto y experiencia. La discapacidad es algo desconocido, algo a lo que no se está acostumbrado a relacionarse. En caso de que no se haya trabajado con discapacidades o no se tengan discapacidades en la familia, es fácil que los viejos prejuicios se activen, entonces no se logra ver que tras el aparato del oxígeno hay un niño común, con una vida emotiva común. El niño tiene como otros niños derecho a formular exigencias a sus padres.

Cuando los padres abandonan al niño, es la Tutela de niños la que debe ayudarlo y no le negará la entrada y no le dejará vivir más con la etiqueta de "paciente". Entonces el escándalo del hospital es un hecho, el sistema de salud puede excusarse en que la Tutela de niños ha estado dentro de la causa sin reaccionar.

Cuando recibo la carta de la Tutela de niños, decido que la causa tiene que ir fuera de la provincia de Nordland. En Nordland hay mucha gente que ha sido envuelta en la causa sin que reaccione. Además, el jefe de las autoridades sanitarias en la provincia de Nordland está casado con una médica en la sección niños. Esta médica también ha sido responsable del equipo del niño.

Envío una carta a las autoridades sanitarias en la provincia NordTrondelag (Nord-Trondelag está al sur de Nordland, es vecina). Explico lo que ha pasado en la causa y escribo que esta causa se trata de un derecho a una vida digna para un niño con discapacidad. Las autoridades sanitarias en la provincia Nord-Trondelag no me comprenden. Ellos no tienen ningún comentario sobre el hecho de que el niño en este tiempo haya vivido ocho años en el hospital. Ellos envían una carta a las Autoridades Sanitarias en la provincia Nordland y les piden estimar una inspección en el piso del niño. 
Las autoridades sanitarias en Nordland visitan al niño en enero de 1998. Esta inspección precedentemente es nombrada bajo el punto IV Vigilancia e inspección.

\section{EL NIÑO Y LA ESCUELA}

El niño está tres años y medio en una escuela normal en Bodo. El primer día en la escuela, en otoño de 1997, el niño viene acompañado de una enfermera y un médico. Sus padres no están allí.

Cuando un hombre tiene una discapacidad para otros es fácil enfocar en la discapacidad, en lo que es distinto. Cuando el niño va la escuela en su silla de ruedas y su tanque de oxígeno, estos ocupan la atención, en lugar del ser humano, en lugar del niño. Mucho más cuando el niño además viene acompañado de un médico y una enfermera, por supuesto reforzando lo "anormal" del niño (Kristiansen 1994) Como sería si el niño hubiera venido andando a la escuela con su tanque de oxígeno dentro de su mochila, acompañado con su madre y su padre. El niño no tiene parálisis en sus piernas. Sin la cánula dentro de su cuello hubiera hablado y ya del primer día sería recibido por amigos.

Ahora la escuela, por primera vez, va a recibir a un alumno que es un paciente. Por supuesto, esto es un reto muy grande para la escuela. En la escuela, hay muchos alumnos con discapacidades. Entre tanto, ellos viven en casa de sus padres. Los padres hacen lo que es posible para que los niños estén lo más integrados posible. Los niños son dejados en la escuela durante el horario escolar y los padres confían en que la escuela puede cuidarles.

En caso del "niño paciente", las circunstancias son totalmente distintas. El primer problema que aparece es que el horario de la escuela no es adecuado al sistema de turnos. La cuestión del equipo es por eso que el niño pueda comenzar más tarde que los otros niños de modo que lo adecúa con el sistema de turnos. A este respecto la escuela consigue imponer su criterio, de modo que el niño pueda comenzar en el mismo tiempo que los otros niños.

Al principio, los profesionales del Hospital exigen estar en la clase (el aula) para cuidarle. A causa de mucho ruido con dos personas del hospital en la clase las personas tienen que estar fuera de la clase. Entonces el Hospital exige que la Escuela ponga a disposición del personal acompañante del niño, una habitación. Porque el niño no tiene que estar sin el cuidado del personal del Hospital. 
Los profesores y la rectora reaccionan a este modo de vivir del niño. Ellos tienen obligación de anunciar a la Tutela de niños de esta situación (es la ley de la Escuela). El problema está ahí: nadie de la escuela anuncia la causa a la Tutela de niños. Aunque la tutela de niños se ha comportado pasivamente, un aviso de la escuela podría haber tenido más eficacia que mi anuncio.

En mi trabajo con esta causa, tomo contacto con la rectora de la escuela. Tenemos una conversación buena. La rectora tiene la misma interpretación de la causa que yo. Ella opina sobre lo malo que es que un niño crezca en un hospital. Sin embargo, no consigue hacer nada. Recibo la comprensión de que ella ha hablado con las autoridades de escuelas en cargos de mayor responsabilidad en el sistema, y que ella ha sido mandada a silenciar.

Cuando un niño está a cargo de una institución pública y ha recibido el cuño de "paciente" en la frente, hay una gran muralla que es imposible de traspasar.

No obstante, es evidente que la rectora, que es un ser humano, quiere fuertemente que el niño consiga una vida mejor. La rectora camina como muchos otros que están envueltos en esta causa y espera a que algunas autoridades alli arriba en el sistema tomen responsabilidad.

\section{LA SEGUNDA CAUSA CRIMINAL Y PENAL, OCTUBRE DE 1997 Y EL DESPIDO}

En junio de 1997, Berthold fue juzgado (bajo el punto VII de la Causa Criminal y Penal). Berthold ha apelado la causa y en octubre de 1997 el Juzgado de Segunda Instancia trata la causa.

La cuestión para el Juzgado es: "En quién vamos a confiar: en Berthold o en los otros del grupo (aquéllos que se quedaron).

El Juzgado confía en el grupo y Berthold es juzgado. La sentencia dice que Berthold de vez en cuando había tomado duramente del brazo al niño, no más. La sentencia no es unánime. Dos conjueces quieren absolverle -el Tribunal está formado por tres jueces y cuatro conjueces-. Durante la causa los jueces están ocupados en las instrucciones sobre el uso del pulmón artificial y el suministro de oxígeno.

La jefa del equipo presenta la nueva instrucción, ésta fue hecha después de que Berthold ya había terminado en el grupo (bajo el punto VII de la Causa Criminal y Penal). Hay mucha polémica sobre la instrucción y el ambiente está irritable. Berthold está desesperado y repite muchas 
veces que él no ha visto esta instrucción antes de que haya aparecido dentro de los documentos de la Causa Criminal y Penal.

La jefa del equipo está sentada, relajada completamente y mira la desesperación de su anterior colega. La jefa no tiene la intención de contar al juzgado la verdad. El.juzğado confía en la jefa del equipo y se fundamenta en que Berthold ha transgredido la instrucción sobre el uso del suministro de oxígeno. La sentencia lleva a que Berthold sea despedido de su puesto como enfermero en el Hospital. Berthold no puede aceptar el despido y habrá una causa sobre este tema también.

Durante la causa laboral mucho es revelado. Aparece que la nueva instrucción fue hecha más de dos meses después de que Berthold ya había terminado en el equipo. Yo pido al Juzgado reanudar la causa criminal y penal. El primero de febrero de 1999, el Tribunal Supremo decide que la causa criminal y penal va a reanudarse.

\section{LA PRIMAVERA DE 1998. EL NIÑO TIENE 9 AÑOS}

En la primavera de 1998 el niño tiene nueve años. ¿Cómo está? El niño es un paciente $y$, por eso, es silencioso. Es imposible conocer sobre lo que pasa con él. Después de que las Autoridades Sanitarias han estado de visita en diciembre de 1997 (bajo el punto IV Vigilancia e Inspección) ningún veedor ha visitado al niño en su piso. No sabemos nada acerca de lo que le ha pasado dentro de su piso. Los que trabajan en el equipo están obligados a mantener el secreto profesional y por eso no dicen nada.

A pesar de que el niño es silencioso y en realidad vive encerrado en su piso, él tiene sus diarios paseos fuera del piso. Cuando el niño y dos o tres personas del equipo están fuera del piso, ellos deben percibir que el niño ha pasado mucho tiempo de su niñez en un centro comercial. Este centro comercial está cerca de su piso. Es fácil tomar un paseo allí para visitar una cafetería o andar por las tiendas. En el verano de 1995 (el período con Berthold) el niño podía usar carrito de apoyo y pasear por sí mismo. Casi tres años después, el niño ha vuelto a su silla de ruedas. La cánula todavía está dentro de su cuello y ésta le impide hablar.

\section{LA GRAN LUCHA}

En el verano de 1998, entro en contacto con Ola Liland. Ola Liland fue educado como pedagogo de la tutela de niños y tiene veinticinco años de experiencia. Trabaja en una oficina que tiene como objetivo 
buscar familias provisionales (bajo el punto IX Mi primera lucha por el niño). Lo especial con Ola Liland es que él es el único empleado de las Autoridades Públicas en la provincia de Nordland que ha tenido valor para salir en los medios y luchar por el niño junto a mí. Él tiene la fuerza psíquica y la integridad que es necesaria para estar en contra del sistema. Para él lo primero es el niño y esto va delante del sistema.

En agosto de 1998, pronuncio un discurso sobre el niño en un seminario en la ciudad de Trondheim. En este seminario representantes del Ministerio de Niños y Familias y también el Ministerio de Sanidad Pública participan. Termino mi discurso con las siguientes palabras:

"Lo único correcto es que las autoridades centrales se pongan en la causa. La causa se trata de un derecho a una vida digna para un niño con discapacidad. En la vida del niño se ven dos caminos: uno es desarrollarse en un oficio, no importa cuál oficio o profesión tenga, sea chofer o algo parecido, u otro camino será desarrollarse como un paciente atendido constantemente.

Yo hablo con un director general adjunto del Ministerio de niños y familias y un director general adjunto del Ministerio de Sanidad Pública. El resultado es que ellos me piden escribir una carta a los Ministerios. Los participantes en el seminario me recomiendan salir en los medios. Muchos de ellos opinan que una causa como ésta no puede solucionarse sin que los medios abran el camino.

Escribo cartas a los dos Ministerios. Lo que pasa es que el Ministerio de Niños y Familias envía una carta al Gobierno Civil de Nordland y el Ministerio de Sanidad Pública envía una carta a las autoridades Sanitarias de la Provincia de Nord-Trondelag (el Jefe de las autoridades sanitarias en la provincia de Nordland es inhábil). Los ministerios piden que la causa sea evaluada de nuevo.

Las Autoridades Centrales retornan la causa a las Autoridades que nunca han opinado que sea malo para un niño crecer en un hospital. El niño es una pelota que está tirada adelante y atrás. Nuestro sistema burocrático es así que las Autoridades Centrales no pueden entrometerse en la autonomía del Municipio y de la Provincia. El Gobierno Civil de Nordland apoya la resolución de la tutela de niños municipal (bajo el punto IX Mi primera lucha por el niño).

El Gobierno Civil tiene su fundamento en nuestra Ley de Tutela de niños para abordar la causa. Cuando el Gobierno Civil no puede ver que es un problema para un niño crecer en un hospital, es poca la ayuda que se puede hacer con las leyes. Los dos trabajadores que tratan la causa del Gobierno Civil tienen educación como trabajadoras sociales. 
Las Autoridades Sanitarias de la provincia de Nord-Trondelag llevan más o menos un año sobre la causa. Durante este tiempo ellos no encuentran ninguna razón para visitar al niño en Bodo. Para ellos es suficiente leer documentos.

Contacto con un periodista en el otoño de 1998. Este periodista escribe mucho sobre la causa. Él entrevista, entre otros, a Ola Liland, a una famosa psicóloga y al defensor de los niños.

Ola Liland manifiesta entre otras cosas: "La cuestión es sobre si la enfermedad va a dirigir la vida del niño o sobre si la vida va a adaptarse a la enfermedad. Todos los niños tienen exigencias en una casa. El niño no tiene una casa. Él se relaciona con numerosas personas, todas ellas están educadas para cuidar a los enfermos".

La psicóloga de niños manifiesta: "Todos se dan cuenta de qué importante es eso de que los niños crezcan en relaciones estables que ellos frecuentemente estén juntos con los mayores no necesariamente es un problema. Los problemas aparecen cuando hay demasiados mayores con los que ellos van a relacionarse. El alargamiento del mundo de los mayores provocará inestabilidad. Los mayores no son confiables y los niños serán solitarios.

Toda la crítica de medios afecta al Hospital, ahora ellos quieren poner orden en la causa.

El niño ha llegado a una bifurcación nueva en su vida. En realidad han pasado más de siete años desde que la resolución fue tomada sobre que el niño es paciente y va a crecer en el Hospital (bajo el punto II Los primeros años del niño). La cuestión es, por supuesto, que es una posibilidad para el Hospital pensar en el niño como algo más que un paciente.

Yo pretenderé que aquí estamos ante profecías realizadas por sí mismos. Se tiene sus propios pensamientos sobre la persona y se relaciona a esta persona de conformidad con dichos pensamientos. Finalmente la persona se presenta así como se piensa que ella es.

El Hospital piensa sobre el niño como paciente y se relaciona con el niño como paciente, por eso es (se queda) paciente. El niño ha llegado a estar dentro de un círculo vicioso del que él no puede salirse.

El Hospital se siente con un paciente realmente único en su regazo y ellos no saben totalmente cómo lo harán. La vivencia del Hospital de que el niño es un paciente único es por lo tanto correcta. "Lo único" es, sin embargo, no su discapacidad primordial, pero eso es la persona que él ha desarrollado a causa de su crecimiento en un hospital. 
La cuestión es que el Hospital a causa de todo lo que ha pasado desde 1991 está en condiciones de tener pensamientos nuevos Cuando durante nueve años se ha visto en el niño a un paciente y se opina que el niño necesita dos profesionales de salud durante veinticuatro horas día y noche para sobrevivir es por supuesto difícil pensar que esta hiperprotección sea errónea. Tanto el Hospital como la madre, también por supuesto, han llegado a una posición defensiva. Es más importante que nunca demostrar que el niño es un paciente. Por medio de señalar que el niño es un paciente único se puede defender el crecimiento que el niño recibe en el Hospital. En una situación así, es imposible pensar en el niño como un ser humano que más que nunca necesita una familia para crecer. Ahora el Hospital determina que el niño va a salir y va a regresar al municipio de sus padres. Es totalmente evidente que el niño no va a mudarse a la casa de su madre o de su padre. Se va a construir una institución similar como la que el niño tiene en Bodo. La situación del cuidado va a ser similar a la que el niño tiene en Bodo. Eso significa que se va a establecer un equipo nuevo alrededor del niño. Dos años pasarán antes de que el niño se mude al municipio de sus padres. Esto pasa en el cambio de mes de noviembre a diciembre de 2000.

La institución del niño está en la vecindad de la madre. El municipio consigue una vivienda que tiene una ubicación tal que el niño puede ver abajo la casa de su madre. El niño puede caminar delante de la casa de la madre. Él puede ver dentro del salón donde su madre y su gemela viven. A causa de su discapacidad el niño no consigue vivir en esta casa, como de costumbre está fuera de su casa.

Muchas personas importantes se envuelven en esta mudanza. Personas de fuera del Hospital y también personas que son trabajadores sociales. El proyecto tiene un presupuesto de quinientos mil euros por año. En este proceso nadie que se encuentre envuelto en la situación se hace la pregunta: "¿Tal vez es mejor buscar una familia provisional al niño? Esta familia por supuesto recibiría ayuda y sostén de la Administración Pública. La base del niño tenđría que ser una familia y no una institución".

\section{¿Por qué no hacerse esta pregunta?}

Desde mi punto de vista se trata de prejuicios frente a las discapacidades. Mucha gente, también profesionales, no creen que haya familias que quieran dar casa a un niño con discapacidad. Además muchos profesionales tienen lástima de los padres que reciben niños con discapacidades. En esta situación es imposible para los profesionales molestar a los padres con la Tutela de Niños. 
El periodista ya anteriormente mencionado, entrevista al defensor de los niños quien se dedica a la causa.

La oficina del Defensor de los niños está en Oslo. Yo tengo muchas conversaciones telefónicas con esta oficina. Les envío todos los documentos que tengo sobre la causa. Además, viajo a Oslo para discutir la causa con ellos. Espero que el Defensor de los Niños esté en condiciones de ver al niño y hablar en contra del sistema. Desde mi punto de vista el defensor de los niños no estaba en condiciones de hacer algo por el niño. En una de mis cartas al defensor de los niños yo hago la siguiente pregunta: " $¿$ Tiene un niño con discapacidad derecho a crecer en una familia? ¿Tiene un niño con discapacidad derecho a exigir algo de sus padres?"

El Defensor de los Niños nunca estuvo en condiciones de contestar a mis preguntas.

Tres años pasaron antes de que yo recibiera una una declaración del Defensor de los Niños. La carta está fechada el veintiocho de diciembre de 2001. En la carta aparece que lo más importante para el Defensor de los Niños es el hecho de que el niño tenga asegurada una oferta de vivienda. En la carta no hay pensamientos sobre la vida emotiva del niño cuando él vive en su vivienda propia.

\section{LA TERCERA CAUSA CRIMINAL Y PENAL (JUNIO-JULIO DE 1999)}

La causa Criminal y Penal ha sido reanudada (bajo el punto XI la segunda causa criminal y penal). La causa es determinada el veintiocho de junio de 1999.

El Juzgado nombra un médico para aconsejar al propio juzgado. Este médico es un experto en enfermedades de pulmón y especialmente en niños que tienen problemas con sus pulmones. El médico, Stein Otto Mullestad, trabaja en un hospital grande en Oslo. Este médico depone su declaración por escrito el veintiocho de junio. Él está presente durante toda la causa y complementa su declaración por escrito al Juzgado. ¿Qué pasa?

Con el experto en la arena, viene por primera vez un experto y observa lo que ha pasado con el niño y el equipo. El equipo en realidad ha estado completamente sin vigilancia e inspección (bajo el punto IV Vigilancia e Inspección).

Stein Otto Mollestad va a visitar al niño en su piso y habla tanto con él como con los profesionales del equipo. Lo que Stein Otto Mollestad 
revela es que, cuando un ser humano está colocado erróneamente (en un hospital en lugar de en una familia), esta situación errónea poco a poco se convierte en una institución de conservación, tanto médica como social. No hay ningún padre o una familia provisional que esté de pie en las barricadas y la lucha, por lo que el niño en realidad está perdido. Stein Otto Mollestad opina que el niño había recibido un tratamiento erróneo. El niño ha recibido menos entrenamiento físico del necesario. Pero lo peor es que el niño lleva una cánula dentro de su cuello. Esta cánula ocasiona al niño muchos problemas. Problemas para hablar. Problemas para comer. Problemas infecciosos (un objeto, una cosa desconocida dentro de su cuerpo). Stein Otto Mollestad opina que todos los niños, también los niños con discapacidades, tienen derecho a crecer en una familia.

Stein Otto Mollestad da su apoyo completo a Berthold. Durante esta causa todo sobre la instrucción nueva y la inutilización de los "informes diarios" es revelado (bajo el punto VII La causa Criminal y Penal).

Berthold es absuelto totalmente. La sentencia es unánime.

\section{DESPUÉS DE LA ABSOLUCIÓN. EL NIÑO TIENE DIEZ AÑOS}

La sentencia es, por supuesto, una derrota grande tanto para la Fiscalía del Ministerio Público como para el Hospital. Todo lo que es revelado no consigue ninguna consecuencia para los empleados envueltos en la misma, ellos continúan su trabajo como si nada hubiera pasado. "Los sistemas" cierran sus puertas y quieren olvidar todo lo embarazoso que fue revelado.

Berthold recibe una indemnización tanto de la Fiscalía del Ministerio Público como del Hospital. El Hospital se siente presionado para hacer algo con la cánula. Alguien viaja con el niño a un hospital en Bergen. En mayo de 2000, la cánula es quitada del cuello del niño.

Durante la tercera causa hemos recibido todavía más comprensión de lo malo y dañoso que es para un niño crecer en una institución. A pesar de todo no ocurre ningún cambio en los pensamientos de las personas que trabajan con el proyecto del niño sobre su mudanza a una institución nueva. Yo contacto al Gobierno Civil para hablar con los dos trabajadores sociales que ya anteriormente he nombrado (bajo el punto XIII La gran Lucha). Hablo con ellos, con cada uno por separado. A pesar de todo lo que ha pasado hay una imposibilidad, para mi, en que ellas puedan cambiar. Especialmente una de ellas destaca que una madre tiene derecho a una vivienda propia para su niño. 


\section{EL DEFENSOR DEL PUEBLO}

Después de haber tenido conversaciones con las dos trabajadoras sociales del Gobierno Civil, estoy con el ánimo muy bajo. Berthold fue absuelto, pero es inútil cuando el niño todavía vive en el hospital y no ha conseguido una vida mejor.

Entonces, hay sólo una única instancia en Noruega que pueda ayudar al niño, el Defensor del Pueblo. Envío toda la causa al Defensor del Pueblo. Mi carta está fechada el nueve de diciembre de 1999. El Defensor del pueblo aborda la causa. Él lleva un tiempo largo sobre la causa. El veintisiete de octubre de 2000, recibo una carta del Defensor del Pueblo. Él escribe que ha escrito al municipio donde el niño va a mudarse y al Gobierno Civil en Nordland. Él les señala que es importante que ahora se emprenda una estimación de la Tutela de Niños.

Después de tres años y medio de lucha, el Defensor del Pueblo me da la razón. El niño con discapacidad tiene derechos frente a su madre y a su padre. El niño con discapacidad es un ser humano.

El Defensor del Pueblo hace una manifestación. Él no puede tomar resoluciones.

Después de la manifestación del Defensor del Pueblo, recibo una carta del Gobierno Civil en Nordland. El contenido de la carta es muy desagradable. Entonces, cuando recibo esta carta, comprendo que la lucha está perdida. La manifestación del Defensor del Pueblo no puede cambiar el primordial entendimiento del Gobierno Civil quien va a seguir la vigilancia en la vivienda del niño.

El niño se muda durante el cambio de mes de noviembre a diciembre de 2000 a su vivienda propia. El niño tiene que relacionarse con un equipo nuevo.

\section{CONCLUSIÓN}

Supongo que la mayoría de la gente opina que el niño vive una trágica fatalidad. Desde mi punto de vista, es culpa de que las principales personas en mi causa tienen lástima a los padres que reciben un niño con discapacidad. En esta situación, las personas principales comprenden y apoyan a aquellos padres que no pueden cuidar a sus hijos con discapacidad. Al mismo tiempo, las personas principales no comprenden que haya gente que quisiera dar una casa a un niño.con discapacidad. En esta situación, es difícil ver que el niño con discapacidad en primer lugar es un ser humano. 
Hablamos sobre prejuicios frente a las discapacidades. Desde mi punto de vista. En caso de que el niño en mi causa hubiera crecido en una familia normal y hubiera recibido tratamiento correcto, el niño hoy caminaría a la escuela con sus botellas de oxígeno en la mochila.

A causa de su crecimiento como paciente el niño se quedará permanentemente paciente.

\section{BIBLIOGRAFÍA}

FOUCAULT, M.: La historia de la locura. Oslo. Gyldendal 1973.

GOFFMAN, E.: Institución y ser humano. Copenhague, Paludan 1993.

GRUE, L.: Familias acostumbradas a niños distintos. Oslo. Ad. Notam. Gyldendal 1993.

KRISTIANSEN, K.: Normalización y estimación del papel social. Oslo. Kommuneforlaget 1994.

SLETTEBA, A.: Asistencia médica y ética. Ad. Notam. Gyldendal 1997.

ONU: Artículos estándar para iguales posibilidades para personas con discapacidad.

ESTADO NORUEGO: Análisis del Estado Noruego sobre condiciones de vida de las personas con discapacidad intelectual 1973 y 1985. 